\title{
From Deficits to Possibilities
}

\author{
Mentoring Lessons from Plants on Cultivating Individual \\ Growth through Environmental Assessment and Optimization
}

Beronda L. Montgomery

\section{Abstract}

The specific ways in which humans engage with the plants growing in their environment offer many lessons about mentoring and professional development interventions. Organisms, such as plants, which largely live out their lives in one location, are exquisitely sensitive to changes in their external environment and adapt their growth to environmental cues to increase survival and productivity. Plants maximize their use and acquisition of available resources and limit or ward off danger from harmful factors. Systematic assessment of how plants sense and respond to environmental fluctuations or transitions, as well as the care humans offer to plants, yield key lessons that can inform mentoring practices that promote the sense-driven and mentor-facilitated success of students and colleagues in academic environments. Notably, the relationships between humans and plants offer inspiration for anticipating and employing specific means of nurturing the success of our students and colleagues. This article discusses plant biology-inspired practices for supporting the comprehensive development of a diverse range of students, academic staff, and faculty members as researchers, scholarly thinkers, and independent practitioners. Ultimately, the growth-perspective relationships with plants that humans regularly exhibit indicate vast potential for our capacity for progressive support of diverse individuals in the academy. In this essay, I investigate effective means for planting and cultivating growth-focused mentoring and faculty development initiatives from a consideration of the intersecting perspectives of plant biology and mentoring.

Many have experienced encountering a plant in an office or a home that is clearly in distress. Wilting leaves or discoloration are clear signs that something has gone wrong. Almost everyone recognizes that a wilting plant means the caretaker has neglected to provide sufficient water. Keen plant caretakers or avid gardeners also often notice the yellowing of leaves as a potential signal of nutrient deficiency and a need for supplemental nutrients such as those in fertilizers. Additionally, nearly all of us have had a plant in our home that bends towards a window. Even when we are savvy enough to turn the plant to redirect its bending, we rarely stop to ponder that this is an active adaptation behavior on the part of plants seeking out light. Plants need light, which drives the production of food in the form of sugars that support plants' growth and fitness, and they will bend themselves to get it. Human responses to plants indicate that many of us are good at reading plants' signals in order to adjust our care of them.

Other contexts, such as learning or collegial environments, do not readily evoke our human responses to plants, which are largely predicated on a mindset or expectation that we can support the growth of individuals or communities through careful cultivation and stewardship of the environment. In learning environments with students or in relationships with colleagues, we often readily ascribe struggles of individuals to deficits possessed by the person or personal failure to thrive. Translating our responses to plants to the care of students and colleagues through intentional mentoring holds great promise for moving from seeing deficits and failures to supporting and enabling possibilities.

Public Philosophy Journal | Volume 1, Number 1 | Spring 2018 


\section{Mentoring as Environmental Stewardship in Mentoring Exchanges}

Others have emphasized how mentoring provides critical support for the development of skills, as well as various forms of care, including socioemotional and psychosocial support, that promote personal and career advancement and success.' Frequently, mentoring entails a hierarchical, and often one-way, flow of information from a senior, experienced individual (i.e., the mentor) to an individual in need of development (i.e., the mentee). ${ }^{2}$ However, mentoring can also be accomplished effectively (and in many cases preferably) by a mentee connected to several individuals in a mentoring network maintained by bilateral exchanges. ${ }^{3}$ At the core of these bilateral exchanges is the reciprocal engagement of individuals in a process, in essence the relationships connecting individuals. Top-down, hierarchical mentoring and network-based mentoring have significant implications for promoting short-term or long-term career advancement, respectively. ${ }^{4}$

Despite the clear outcomes associated with mentoring, it frequently focuses on building up deficits in individual mentees, rather than promoting the growth of inexperienced, yet otherwise capable, individuals. ${ }^{5}$ In particular, primarily white or majority institutions commonly adopt individual-deficit mentoring models in attempting to 'support' individuals in adapting to academic environments, particularly in regards to minoritized or underrepresented students and faculty. ${ }^{6}$ Broadly implemented, a deficits-focused approach centers on a fixed mindset perspective, or one that presumes innate, fixed potential. Less frequently do institutions attempt to investigate or assess the effects of prevailing and commonly accepted biases, institutional cultures, or structural barriers, such as racism, sexism, or classism, present in academic environments in which students or faculty exhibit challenges progressing towards intended individual outcomes. Culture-centered or barrier-mediating interventions depend upon surveying environments comprehensively and initiating active efforts to position institutions to address barriers across entire ecosystems, not just defaulting to a unilateral focus on fixing individual-related concerns. ${ }^{7}$ These approaches embrace and promote a growth mindset rather than the fixed mindset perspective. ${ }^{8}$

The unilateral focus on individual deficits that underlies 'fixed mindset' perspectives will continue to result in the limited impacts and meager institutional outcomes and growth we have observed for years. Broadening participation efforts in learning and professional environments or gender equity initiatives have stalled, such that participants fail to fully reflect national demographics. Further, 'fixed mindset' interventions fail to fully promote innovation. ${ }^{9}$ Mentors and leaders have critical roles in promoting growth-focused engagement based on the potential of individuals on a day-to-day basis. Acknowledging the need to address the environments in which mentoring occurs is critical and urgent. To be effective, these approaches require centering mentees and their individual potential for growth in mentoring exchanges and outcomes. We must also understand that facilitating best outcomes requires extensive consideration of ecosystem contexts and the potential roles mentors and leaders have in promoting attentiveness to colleagues and cultivating cultures based on the expectation of genuine collegiality, support, and care.

\section{Decentering the Mentor in Mentoring Exchanges}

Mentoring is based on personal relationships that support the growth of an individual being mentored in myriad ways. ${ }^{10}$ Effective mentoring includes skills development and psychosocial or socioemotional support designed
1. See Dana Haggard et al., "Who Is a Mentor? A Review of Evolving Definitions and Implications for Research," Journal of Management 37, no. 1 (2011): 280-

304; Maryann Jacobi, "Mentoring and Undergraduate Academic Success: A Literature Review," Review of Educational Research 61, no. 4 (1991): 505-32; Kathy E. Kram, Mentoring at Work: Developmental Relationships in Organizational Life (Glenview, IL: Scott, Foresman, 1985); and Becky Wai-Ling Packard, Successful STEM Mentoring Initiatives for Underrepresented Students: A Research-Based Guide for Faculty and Administrators (Sterling, VA: Stylus Publishing, 2016).

2. See Beronda Montgomery, "Mapping a Mentoring Roadmap and Developing a Supportive Network for Strategic Career Advancement," SAGE Open 7, no. 2 (2017): 1-13, https://doi.org/10.1177/2158244017710288.

3. See Monica C. Higgins and Kathy E. Kram, "Reconceptualizing Mentoring at Work: A Developmental Network Perspective," Academy of Management Review 26, no. 2 (2001): 264-88; Montgomery, "Mapping"; Kerry A. Rockquemore, "A New Model of Mentoring," Inside Higher Ed, July 22, 2013, https://www.insidehighered.com/ advice/2013/07/22/essay-calling-senior-faculty embrace-new-style-mentoring; and Mary Deane Sorcinelli and Jung Yun, "From Mentor to Mentoring Networks: Mentoring in the New Academy," Change: The Magazine of Higher Learning 39, no. 6 (2007): 58-61.

4. See Monica C. Higgins and David A. Thomas, "Constellations and Careers: Toward Understanding the Effects of Multiple Developmental Relationships," Journal of Organizational Behavior 22, no. 3 (2001): 223-47.

5. See Shaun R. Harper, "An Anti-Deficit Achievement Framework for Research on Students of Color in STEM," New Directions for Institutional Research 148 (2010): 63-74, https://dx.doi.org/10.1002/ir.362.

6. See Harper, "Anti-Deficit"; Packard Successful STEM; and Joseph A. Whittaker, Beronda L. Montgomery, et al., "Retention of Underrepresented Minority Faculty: Strategic Initiatives for Institutional Value tProposition Based on Perspectives from a Range of Academic Institutions," Journal of Undergraduate Neuroscience Education 13 no. 3 (2015): A136-A145

7. See Beronda L. Montgomery, "Pathways to Transformation: Institutional Innovation for Promoting Progressive Mentoring and Advancement in Higher Education," Susan Bulkeley Butler Center for Leadership Excellence and ADVANCE Working Paper Series 1, no. 1 (2018): 10-18.

8. See Carol Dweck, "What Having a 'Growth Mindset' Actually Means," Harvard Business Review, January 13, 2016, http://hbr org/2016/01/what-having-a-growth-mindsetactually-means. Accessed June 6, 2018.

9. See Joseph A. Whittaker and Beronda L. Montgomery, "Cultivating Diversity and Competency in STEM: Challenges and Remedies for Removing Virtual Barriers to Constructing Diverse Higher Education Communities of Success," Journal of Undergraduate Neuroscience Education 11, no. 1 (2012): A44-A51

10. See Montgomery, "Mapping." 
to lead to career advancement and success." Most traditional and prevalent mentoring paradigms, which are intended to support mentees in moving ahead in academic, personal, and/or career goals, tend, in practice, to focus on the mentors and their values (i.e., affirming mentor views of valid developmental or career paths, or maintaining status quo views of success in particular disciplines or institutions). This widely accepted view of mentoring can de-center individual mentees' self-defined goals and views of success, and, if 'successful,' can actually limit individuals' unique contributions to communities. Truly supportive mentors could help prepare and develop individual views, goals, and trajectories with an understanding of and input on promoting contextual, community success. This idea of contextual success draws clear parallels to plant care. In this regard, mentors promote success by facilitating individual progress or advancement in a particular environment. In the case of supporting colleagues in the pursuit of self-defined goals, mentors and leaders can promote habits of goals setting and of establishing expectations for broad success among diverse individuals in particular contexts. Ultimately, mentoring will improve and be more successful when it is clear that excellent mentoring is not really about the mentor. Excellent mentoring requires that mentors support individual mentees as they move along a self-defined path in a particular context.12

\section{Human Capacity for Facilitating Growth-Mindset Based Support and Individual Development}

Mentors and leaders in many domains, from education to the workforce, have increasingly recognized the importance of cultivating a 'growth mindset,' or the belief that the talents and abilities of individuals can be developed or cultivated. Largely attributed to psychologist Carol Dweck, the growth mindset paradigm stands in contrast to the 'fixed mindset' perspective, which is based on a belief in the innate abilities of individuals. Whereas many individuals strongly support the idea of cultivating a culture based on the growth mindset, others struggle with the tendency to overgeneralize the concept, as well as with how to develop specific and effective means of implementation. ${ }^{3}$ Additionally, institutional review and reward structures can actually impede the promotion of reciprocity and colleague care, as the prevailing focus on advancement reinforces self-promotion and competition, as well as models of individual success. ${ }^{14}$ One particular challenge is whether a growth-mindset culture means encouraging individuals to exhibit resilience or adaptation and the associated expectations that they can grow in a 'static environment.' However, the relationship between humans and plants teaches us that a growth mindset is indeed our default human mode of caring for plants.

A complementary approach to the growth mindset orientation is appreciative inquiry, ${ }^{15}$ a concept that engages strengths as a starting place for promoting individual change and growth. Diana Whitney and David Cooperrider have described appreciative inquiry as "affirming past and present strengths, successes, and potentials."16 Also central to this concept is the expectation "that every organization and community"-and I would add individual - "has many untapped and rich accounts of the positive."17 The appreciative inquiry approach has potential to counteract deficits framing and its associated limitations, such as its "unfortunate propensity to reinforce hierarchy" rather than promote broad-based access. ${ }^{18}$ Framing mentoring according to appreciative-inquiry tightly links strengths-based engagement of mentees with an ability to recognize a range of cultural and navigational capital as foundations from which to launch success. ${ }^{19}$
11. See Haggard et al., "Who is a Mentor?"; Jacobi, "Mentoring"; Kathy E. Kram, "Phases of the Mentor Relationship," Academy of Management Journal 26, no. 4 (1983): 608-25; and Packard, SUCcessful STEM.

12. See Montgomery, "Mapping".

13. See Dweck, "Growth Mindset".

14. See A. Casadevall and F. C. Fang, "Reforming Science: Methodology and Cultural Reforms," Infection and Immunity 80 (2015): 897-96; and Beronda L. Montgomery et al., "Guiding the Way: Mentoring Graduate Students and Junior Faculty for Sustainable Academic Careers," SACE Open 4, no. 4 (2014): 1-11, https://doi.org/10.1177/2158244014558043.

15. Appreciative Inquiry Commons, https://appreciativeinquiry.champlain edul.

16. Diana Whitney and David Cooperrider, Appreciative Inquiry: A Positive Revolution in Change (San Frnacisco, CA Berrett-Koehler Publishers, Inc., 2005), 9.

17. Whitney and Cooperrider, Appreciative Inquiry, 10

18. Whitney and Cooperrider Appreciative Inquiry, 3 .

19. Torie Weiston-Serdan, Critical Mentoring: A Practical Cuide (Sterling, VA: Stylus Publishing, 2017), 66 
Readily observed human engagement with plants aligns well with both growth mindset and appreciative inquiry approaches. When a plant is not faring well in its environment, caretakers ask a multitude of questions about environmental factors (light, water, temperature, nutrients, etc.) that may be suboptimal to support the health and success of the individual organism (Figure 1A). This response is generally distinct from our response to other humans, which frequently highlights presumed weaknesses and deficits in the individual rather than suboptimal environmental factors. Our human responses to plants in our environment provide an undeniable proxy for how we can apply a growth or appreciative inquiry-based mindset rather than a fixed mindset to mentoring relationships. A comprehensive growth mindset-based approach to mentoring or individual development cultivates a bilateral focus on individuals and environmental contributors in promoting success of individuals. Fortunately, in some universities and community-based outreach/engagement programs, as well as with some individual mentoring programs or comprehensive student success or faculty development initiatives, mentors and leaders are beginning to question the environmental impacts on individual potential for success or growth.

Figure 1. What mentoring lessons arise from thoughtful assessment of humans' care of plants in shared environments?

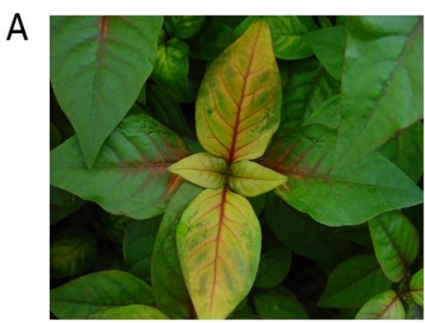

What do we generally do when our plants are not faring well?

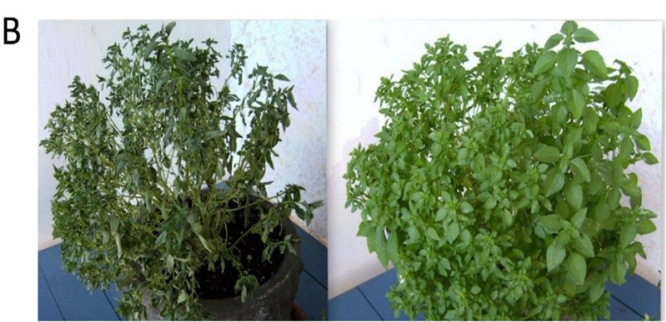

unwatered plant

watered plant

(A) Human responses to plants that are not faring well include extensively probing the environmental factors that may be contributing to failure to thrive rather than defaulting to an individual-deficit causation. (B) Sometimes supporting growth requires relocating resources present in the environment to proximity of individuals to support growth. For example, relocated water from faucet to the soil in which a plant is growing results in a healthy watered plant, compared to a similar unwatered plant that does not have access to the resource. Photos from public domain, photos in panel B by Victor M. Vicente Selvas.

\section{Individual-Environment Interactions}

In addition to assessing growth, development, and progress from the perspective of each individual, there are specific environmental factors that deserve attention when assessing the potential for individual success. Unfortunately, in direct departure from our common responses to plants in our environments, we often hesitate to ask questions about the impact of one's environment on supporting or hampering individual learning and career success. Indeed, the most common response a caretaker has when a plant exhibits impaired or less than optimal growth is to first and foremost begin with an assessment of the plant's environment. Frequently asked questions include whether the plant is receiving enough or too much light; whether the nutrients needed to sustain plant life are available, accessible, and sufficient; whether the plant is being watered adequately; and whether pests or herbivores are causing life-threatening damage or reduced fitness of the plant. Caretakers generally conduct a thorough analysis of the potential im- 
pacts of the living and non-living components of a plant's environment. Typically, additional interventions and subsequent valuations of the applied mediations follow this assessment to assure that the attempts to make things better for the individual plant are actually working.

In the event that a plant remains challenged in growth, caretakers commonly determine that the problem lies with their caretaking skills. For example, we frequently hear individuals declaring that they simply do not have a "green thumb." Whether the plant has a genetic defect or is incapable of growing successfully in its environment (i.e., attributes of a deficit state) are generally the last types of questions that we ask about a plant that is not surviving or thriving in its environment. With other humans, however, we often reverse this series of questions. We can be quick to recognize weaknesses or judge individuals as incapable of growth or progress when challenges arise. We often default to such responses rather than asking bilateral questions about the individual and the environment in which the individual exists. This is particularly prevalent with students, especially those from groups underrepresented in the academy, and with junior faculty, again likely those from groups underrepresented in their disciplines and institutions. When such minoritized or underrepresented individuals encounter challenges acclimating to or succeeding in particular environments, the system often labels them 'unable to succeed.' Those in a position to judge presume the environment is largely innocent of detrimental impacts, or of containing 'environmental barriers' to success, ${ }^{20}$ which indeed may be limiting the potential of the individual. We need to invoke our natural human responses to plants to probe many aspects of the environment, as well as the efficacy (or lack thereof) of our responsive tending of the environment as critical for broadly promoting success.

\section{Plant Growth-Inspired Mentoring Implications}

To cultivate individual growth using growth-promoting perspectives drawn from successful cultivation of plants, specific human responses to plants should inspire effective leadership and mentoring approaches. Here, I consider specific environmental parameters associated with the effective growth of individuals and discuss effective means for applying these parameters to supporting individual students and faculty members from diverse backgrounds. Specifically, I suggest mentoring lessons that should be considered across a range of contexts, as well as appropriate measures that can be enacted.

\section{Plant Lesson 7}

First, we extensively probe our environment when the plants in it are not faring well.

\section{Mentoring Implication 7}

Instead of beginning with questions about personal deficits, this lesson from plants teaches that our mentoring engagements with students and junior colleagues should begin with asking systematic questions about the impacts of the environment on an individual's potential for growth and success. We should not by default presume infallibility of the system. A well-informed understanding of the system's impact on the individual will greatly enrich and elevate our mentoring practices.

\section{Plant Lesson 2}

We recognize that successful plant growth sometimes requires new resources and at other times the relocation of existing resources.
20. See Whittaker and Montgomery "Cultivating Diversity". 
When we have identified specific environmental deficits or needs, we assess whether new resources are needed or whether resources already present somewhere in the ecosystem need to be relocated and/or connected to the individual to support its growth and development. Sometimes, by default, we assume that growth requires new resources, which can readily become an impediment to progress or innovation in resource-limited environments. While some situations might require new resources, we might simply need to recognize and relocate pre-existing resources. For example, water in a faucet is present in the environment, but it is of absolutely no use to a plant until it is relocated from the faucet to the soil in which a plant is growing (Figure 1B). Well-cultivated knowledge of which resources exist in a particular environment, combined with full awareness of individuals' needs, allows mentors to make targeted efforts to connect students and colleagues with resources to help them succeed.

In these instances, one of the mentor's primary and significant roles is to serve as an environmental steward who connects individuals to salient resources.

In other cases, available resources of the right "type" might prove insufficient to support the maximal growth of an individual in a particular context. For example, tap water from the faucet might be substandard to purified water due to impurities or other issues. Thus, an available but insufficient resource does not yield the desired growth. Here, a growth-minded mentor would recognize when available resources are insufficient and facilitate connection to suitable alternatives or assist in altering an existing resource (e.g., equivalent to identifying filters to purify polluted tap water). Leaders may facilitate such transformation of resources by providing developmental support or training to improve mentoring in a department. Leaders also have key roles in establishing an expectation that colleagues serve in such supportive roles and rewarding accordingly.

The necessary resources are not always already present in any given environment. For instance, extending our example of supporting plant growth, entirely new resources such as bottled water or fertilizers rather than mere tap or filtered water may be needed. A situation like this presents an opportunity for a mentor or leader to help identify and acquire new resources. The various roles of mentors in identifying and accessing resources positions them as "opportunity brokers" who can help individuals connect to resources, spanning from practical to additional mentoring, to promote their success. ${ }^{21}$

\section{Plant Lesson 3}

We recognize that caretakers and their specific preparation, expertise, and efficacy in using these skills, critically matter to plant persistence and optimized survival.

\section{Mentoring Implication 3}

Given two plants with equal potential for growth, the one best connected to available and sufficient resources will grow better and exhibit greater productivity than the plant with equal potential but limited or insufficient access to the necessary resources. The ability of a caretaker to recognize the plants' current and evolving needs and to both identify and make connections to required resources is critical in supporting growth and potential. Likewise, mentors and leaders greatly matter in supporting the success of individual mentees. Given two individuals of equal aptitude, the one connected to the right mentoring resources or imbedded in the right mentoring network is much more likely to succeed. The potential for positive mentoring outcomes
21. Weiston-Serdan, Critical Mentoring, 47; see also Montgomery, "Mapping". 
and individual success depends, in large part, on the caretakers in established mentoring networks recognizing how their own experience, expertise, and access to resources can serve their mentees' needs and advance their goals. To effectively do so requires prioritizing the assessment of care between students and faculty instructors, or among colleagues. Leaders can set intentional and explicit goals, as well as provide protected time and incentives to those individuals who seek to elevate their care of colleagues and stewardship of communities.

\section{Plant Lesson 4}

We seek external expertise when our own caretaking efforts prove ineffective or when we lack knowledge about the underlying causes of the impairments or limitations in plant growth.

\section{Mentoring Implication 4}

When plants are not growing well, we will often seek the assistance of someone we know to be "good" at growing plants for advice or assistance. That is, we actively seek "mentoring" or "coaching" to serve as better caretakers, including seeking advice on identifying resources to which the plant may need access or specific advice on means for us to provide better care. When mentoring relationships are not progressing well, mentors and leaders can likewise seek external advice from others with experience, or mentors can seek training to improve their mentoring. ${ }^{22}$ Such advice may center on specific mentoring actions to take, on specific assistance in increasing mentor awareness of resources, or facilitating connections of those mentored to available resources. ${ }^{23}$ Just as it is not seen as a weakness to seek input on plant care practices from those who have more experience, expertise, or success in caring for these organisms, we need to promote environments and cultures in which seeking external advice about mentoring is seen as a strength, indeed a responsibility, which is actively encouraged, recognized, and rewarded.

\section{Plant Lesson 5}

We attribute a failure to support plant growth to our own caretaking and stewardship inadequacies or inabilities and seek opportunities to improve our care regimen.

\section{Mentoring Implication 5}

In the unfortunate event that a mentoring relationship or exchange is not progressing towards successful guidance of an individual student or junior faculty member, the care humans offer plants teaches us to consider that the mentor may not be meeting the needs of the individual rather than an individual mentee having intractable deficits. Certainly, individual plant caretakers frequently admit to "not having a green thumb." In the case of a "care failure," caretakers may allow a plant to be cared for by another who demonstrates the ability to successfully complete the task. This sort of transfer happens much less frequently in the mentoring realm, even though it may be closely linked to increasing retention of mentees with diverse levels of experience or preparation, or in cases where cultural or other mismatches might impede success in a mentoring exchange. In the event of mismatches related to culture, in particular, emergent interventions related to "culturally relevant practice" in mentoring can potentially improve mentors' abilities to support a range of individuals from diverse backgrounds. ${ }^{24}$ Specifically, such culturally-relevant practices can "help us to understand how we can focus on and leverage the wealth of the culture in marginalized and minoritized communities," in addition to assessing systems-based structural barriers that impede processes. ${ }^{25}$ In cases where a particular mentor is not serving well for
22. See Angela M. Byars-Winston et al., "Culturally Diverse Undergraduate Researchers' Academic Outcomes and Perceptions of Their Research Mentoring Relationships," International Journal of Science Education 37, no. 15 (2015): 2533-53; Christine Pfund et al., "The Merits of Training Mentors," Science 311, no. 5760 (2006): 473-74; Christine Pfund et al., "Training Mentors of Clinical and Translational Research Scholars: A Randomized Controlled Trial," Academic Medicine 89, no. 5 (2014): 774-82; Christine Pfund et al., "Building National Capacity for Research Mentor Training: An EvidenceBased Approach to Training the Trainers," CBE-Life Sciences Education 14, no. 2 (2015): ar24, https://doi.org/10.1187/cbe.1410-0184.

23. Center for the Improvement of Mentored Experiences in Research, https:/l cimerproject.org/\#/; National Research Mentoring Network, https://nrmnet. net/; https://commons.mtholyoke.edu/ beckypackard/resources/.

24. Weiston-Serdan, Critical Mentoring, 44; see also Angela Byars-Winston, "Toward a Framework for Multicultural STEMFocused Career Interventions," Career Development Quarterly 62, no. 4 (2014): 340-57.

25. Weiston-Serdan, Critical Mentoring, 44; see also Whittaker and Montgomery, "Cultivating Diversity". 
the mentee, and perhaps the mentor has already sought external advice in improving the mentoring exchange, it should not be perceived as a failure to admit that the situation is not likely to end well and to actively, and in a timely fashion, facilitate transfer of the individual to a more suitable caretaker. To do otherwise (i.e., engage with a mentee for whom one's specific mentoring skills are not well matched), could lead to harm or complete failure to thrive.26 In all cases, the focus should be on supporting growth; thus, doing no harm, independent of intentions.

We must address the prevalent academic culture of outright ignoring "care failures," or even worse tolerating them as long as the ill-effective mentor remains a "productive" scholar. Attracting recognized accolades or financial support, or prolifically generating "high impact" scholarly outcomes such as peer-reviewed publications should not excuse ill-effective mentoring. In this regard, leaders have opportunities (and arguably responsibilities) to establish and cultivate environments that expect, encourage, and specifically empower effective colleague care.

\section{Plant Lesson 6}

As a very last resort, when a plant is not faring well, we may attribute this outcome to a failure of a plant to thrive.

\section{Mentoring Implication 6}

In cases where a caretaker has exhausted all environmental interventions, has sought training to improve potential care outcomes, or has unsuccessfully called on another to intervene, the individual may ultimately reach a decision that the plant exhibits deficits or is incapable of thriving in a particular environment. However, in such instances, there is often no real negative judgement of the plant per se but often a reluctant acceptance of failure to support its growth. Mentors and leaders need to cultivate such a perspective for individuals seeking support for success in their environments. A failure to realize success in one environment or with a particular set of caretakers does not necessarily mean that an individual cannot be successful elsewhere. Ultimately, attempting to reflect on processes in a manner that presumes the potential for growth of all individuals can keep open the possibility that the right set of conditions, environmental factors, and caretakers could support an individual's success. Such a perspective can leave the individual seeking impactful mentoring input heartened rather than completely demoralized in regards to potential alternatives. This perspective leaves us pursuing non-deficit- or appreciative-inquiry-based mentoring, which "becomes much more about interrogating context and acting based on critical analysis of that context, rather than an immotile relationship reinforced by hierarchy and saviorism." 27

\section{Perspective}

There is a wealth of knowledge and mentoring inspiration to be derived from observing, contemplating, and enacting lessons from the care that humans offer to plants in a shared space. Our growth-perspective and appreciative-inquiry driven engagement with plants can help transform the experiences of students and junior colleagues who are being mentored towards attainment of personal and professional goals. Admittedly, there are limits to analogies such as the plant-based one presented here, including that there are many impediments to implementing effective mentoring in many environments. Such impediments include limited knowledge and training, minimal rewards associated with effective mentoring, limited structures of assessment and accountability, and prevalent academic cultures which fail to reward growth-based, care-centered approaches to engagements with colleagues. Our environments consistently prioritize individualized mod-
26. Weiston-Serdan, Critical Mentoring, 59. 27. Weiston-Serdan, Critical Mentoring, 1-2. 
els of success over community-based collegiality and reciprocity. However, where effectively enacted, pivoting from a deficit-based approach to mentoring, especially of individuals from groups underrepresented in academic and professional environments, to a growth-based mentoring approach has great potential for significantly increasing the retention of individuals we recruit to these spaces. Furthermore, the existence and thriving of these individuals in our environment not only supports their growth but also allows their presence to positively impact our communities. We have to learn to "read" and observe the growth cues of colleagues in our environments as evidence of their health, or lack thereof, in context. We, then, must use these cues-much as we do with plants - to guide our care of colleagues to support their success and thriving.

Michigan State University

\section{BIBLIOGRAPHY}

Byars-Winston, Angela. "Toward a Framework for Multicultural STEM-Focused Career Interventions." Career Development Quarterly 62, no. 4 (2014): 340-57.

Byars-Winston, Angela M., Janet Branchaw, Christine Pfund, Patrice Leverett, and Joseph Newton. "Culturally Diverse Undergraduate Researchers' Academic Outcomes and Perceptions of Their Research Mentoring Relationships." International Journal of Science Education 37, no. 15 (2015): 2533-54.

Casadevall, A., and Fang, F. C. "Reforming Science: Methodological and Cultural Reforms." Infection and Immunity 80 (2015): 891-96.

Dweck, Carol. "What Having a 'Growth Mindset' Actually Means." Harvard Business Review. January 13, 2016. https://hbr.org/2016/01/what-havinga-growth-mindset-actually-means. Accessed June 6, 2018.

Haggard, Dana L., Thomas W. Dougherty, Daniel B. Turban, and James E. Wilbanks. "Who Is a Mentor? A Review of Evolving Definitions and Implications for Research." Journal of Management 37, no. 1 (2011): 280-304.

Harper, Shaun R. "An Anti-Deficit Achievement Framework for Research on Students of Color in STEM." New Directions for Institutional Research 148 (2010): 63-74. https://dx.doi.org/10.1002/ir.362.

Higgins, Monica C., and Kathy E. Kram. "Reconceptualizing Mentoring at Work: A Developmental Network Perspective." Academy of Management Review 26, no. 2 (2001): 264-88.

Higgins, Monica C., and David A. Thomas. "Constellations and Careers: Toward Understanding the Effects of Multiple Developmental Relationships." Journal of Organizational Behavior 22, no. 3 (2001): 223-47.

Jacobi, Maryann. "Mentoring and Undergraduate Academic Success: A Literature Review." Review of Educational Research 61, no. 4 (1991): 505-32.

Kram, Kathy E. "Phases of the Mentor Relationship." Academy of Management Journal 26, no. 4 (1983): 608-25.

Kram, Kathy E. Mentoring at Work: Developmental Relationships in Organizational Life. Glenview, IL: Scott, Foresman, 1985.

Montgomery, Beronda L. "Mapping a Mentoring Roadmap and Developing a Supportive Network for Strategic Career Advancement." SAGE Open 7, 
no. 2 (2017): 1-13. https://doi.org/10.1177/2158244017710288.

Montgomery, Beronda L. "Pathways to Transformation: Institutional Innovation for Promoting Progressive Mentoring and Advancement in Higher Education." Susan Bulkeley Butler Center for Leadership Excellence and ADVANCE Working Paper Series 1, no. 1 (2018): 10-18.

Montgomery, Beronda L., Jualynne E. Dodson, and Sonya M. Johnson. "Guiding the Way: Mentoring Graduate Students and Junior Faculty for Sustainable Academic Careers." SAGE Open 4, no. 4 (2014): 1-11. https://doi. org/10.1177/2158244014558043.

Packard, Becky Wai-Ling. Successful STEM Mentoring Initiatives for Underrepresented Students: A Research-Based Guide for Faculty and Administrators. Sterling, VA: Stylus Publishing, 2016.

Pfund, Christine, Stephanie C. House, Pamela Asquith, Michael F. Fleming, Kevin A. Buhr, Ellen L. Burnham, Julie M. Eichenberger Gilmore, et al. "Training Mentors of Clinical and Translational Research Scholars: A Randomized Controlled Trial." Academic Medicine 89, no. 5 (2014): 774-82.

Pfund, Christine, Christine Maidl Pribbenow, Janet Branchaw, Sarah Miller Lauffer, and Jo Handelsman. "The Merits of Training Mentors." Science 311, no. 5760 (2006): 473-74.

Pfund, Christine, Kimberly C. Spencer, Pamela Asquith, Stephanie C. House, Sarah Miller, and Christine A. Sorkness. "Building National Capacity for Research Mentor Training: An Evidence-Based Approach to Training the Trainers." CBE-Life Sciences Education 14, no. 2 (2015): ar24. https://doi. org/70.1187/cbe.14-10-0184.

Rockquemore, Kerry A. "A New Model of Mentoring." Inside Higher Ed. July 22, 2013. https://www.insidehighered.com/advice/2013/07/22/essay-calling-senior-faculty-embrace-new-style-mentoring.

Sorcinelli, Mary Deane, and Jung Yun. "From Mentor to Mentoring Networks: Mentoring in the New Academy." Change: The Magazine of Higher Learning 39, no. 6 (2007): 58-61.

Weiston-Serdan, Torie. Critical Mentoring: A Practical Guide. Sterling, VA: Stylus Publishing, 2017.

Whitney, Diana, and David Cooperrider. Appreciative Inquiry: A Positive Revolution in Change. San Francisco, CA: Berrett-Koehler Publishers, Inc., 2005.

Whittaker, Joseph A., and Beronda L. Montgomery. "Cultivating Diversity and Competency in STEM: Challenges and Remedies for Removing Virtual Barriers to Constructing Diverse Higher Education Communities of Success." Journal of Undergraduate Neuroscience Education 11, no. 1 (2012): A44-A51.

Whittaker, Joseph A., Beronda L. Montgomery, and Veronica G. Martinez Acosta. "Retention of Underrepresented Minority Faculty: Strategic Initiatives for Institutional Value Proposition Based on Perspectives from a Range of Academic Institutions." Journal of Undergraduate Neuroscience Education 13, no. 3 (2015): A136-A145. 


\section{Acknowledgements}

The author thanks Dr. Marquita Qualls, host and producer of the "Beyond the Bench: STEMulating Career Conversations" podcast for reigniting my focus on writing this essay in a recent podcast conversation (https://www.stitcher. $\mathrm{com} /$ podcast/marquita-m-qualls-phd/beyond-the-bench-stemulating-career-conversations/e/51212294). Research in the author's lab on plant growth responses to the environment is supported by the National Science Foundation (grant no. MCB-1515002 to Beronda L. Montgomery). Work on growth responses of photosynthetic organisms and on mentoring is supported by the Michigan State University Foundation.

Beronda L. Montgomery (montg133@msu.edu; @BerondaM) is MSU Foundation Professor in the Departments of Biochemistry \& Molecular Biology and Microbiology \& Molecular Genetics in the Department of Energy_Plant Research Laboratory at Michigan State University. She also serves as assistant provost for Faculty Development-Research. In addition to research in the area of environmental responses of photosynthetic organisms, Dr. Montgomery's practical interests in higher education policy include mentoring, leadership, and increasing diversity in the natural sciences and in the professoriate in general. 


\section{From Deficits to Possibilities}

\section{A Public Holistic Response}

\section{Christopher P. Long}

The power of this essay lies in the extended and textured analogy it establishes and develops between nurturing the growth of plants and nurturing the growth of our academic colleagues. At root, the lesson this essay invites us to learn is that we need to shift our ways of thinking about faculty and student mentoring from a reactionary mindset that focuses on deficits, to a self-reflective growth mindset in which we work together to create an environment in which our colleagues will thrive.

As with any analogy, there are limits to the one developed here. Plants, unlike colleagues, cannot move of their own volition, so they are dependent on their caretakers in a different way. Further, plants communicate by showing, while our colleagues can articulate a vision of meaningful work.

Holding these, and perhaps other differences in mind, however, the lessons we learn from the analogy here are vital to our attempts to nurture the success of our colleagues. Of particular salience in this regard is the orientation toward critical self-reflection Montgomery encourages us to take as we consider how best to create an environment in which our colleagues from traditionally underrepresented minorities will be able to do their best work. This requires us to consider long-standing systems of structural racism and inequity that can only be dismantled when they are assiduously identified and intentionally redressed through strategic and sustained interventions.

Nurturing an enriching environment of care and mindful concern in which the success of our colleagues is seen as an important indicator of our success as a university cuts against the grain of a system of academic metrics that reward self-interest and legitimize the mechanisms of institutional ranking that determine reputational value. The academic community to which this essay is directed urgently needs to learn the habits of the growth mindset mentoring practice for which Montgomery advocates. If, however, we are to change the culture of the academy, a deep environmental intervention is needed that goes to the very roots of how we measure, reward, and advance scholarship that enriches individual and community life.

Michigan State University

Christopher P. Long (cplong@msu.edu) is professor of philosophy and dean of the College of Arts and Letters at Michigan State University. ttHe is co-founder and editor of the Public Philosophy Journal and the editor the Journal for General Education. 